Meta

Journal des traducteurs

Translators' Journal

\title{
Formation à la traduction technique
}

\section{Hugo Marquant}

Volume 50, numéro 1, mars 2005

Enseignement de la traduction dans le monde

Teaching Translation Throughout the World

URI : https://id.erudit.org/iderudit/010663ar

DOI : https://doi.org/10.7202/010663ar

Aller au sommaire du numéro

Éditeur(s)

Les Presses de l'Université de Montréal

ISSN

0026-0452 (imprimé)

1492-1421 (numérique)

Découvrir la revue

Citer cet article

Marquant, H. (2005). Formation à la traduction technique. Meta, 50(1), 129-136. https://doi.org/10.7202/010663ar

\section{Résumé de l'article}

Après avoir analysé la notion de « traduction technique » (1) d'un double point de vue : les « contenus techniques " (1.1) et le " discours technique » (1.2), l'auteur présente quelques principes de méthodologie générale de la " formation à la traduction technique » (2) comme l'« employabilité » (2.1), l'alternance « désarticulation vs approche holistique » (2.2) et la " représentativité du domaine " (2.3).

Pour clôturer, il analyse brièvement quelques éléments de méthodologie concrète (3) comme la dynamique professionnelle (TRADUTECH et STAGEM) (3.1), la visualisation et la compréhension objective (3.2) et le texte/produit (3.3).
Ce document est protégé par la loi sur le droit d'auteur. L'utilisation des services d'Érudit (y compris la reproduction) est assujettie à sa politique d'utilisation que vous pouvez consulter en ligne.

https://apropos.erudit.org/fr/usagers/politique-dutilisation/ 


\title{
Formation à la traduction technique
}

\author{
HUGO MARQUANT \\ Institut Libre Marie Haps - Haute École Léonard de Vinci, Bruxelles, Belgique \\ hugo.marquant@vinci.be
}

\begin{abstract}
RÉSUMÉ
Après avoir analysé la notion de «traduction technique» (1) d'un double point de vue: les «contenus techniques» (1.1) et le «discours technique» (1.2), l'auteur présente quelques principes de méthodologie générale de la «formation à la traduction technique» (2) comme l'«employabilité» (2.1), l'alternance «désarticulation vs approche holistique» (2.2) et la «représentativité du domaine» (2.3).

Pour clôturer, il analyse brièvement quelques éléments de méthodologie concrète (3) comme la dynamique professionnelle (TRADUTECH et STAGEM) (3.1), la visualisation et la compréhension objective (3.2) et le texte/produit (3.3).
\end{abstract}

\section{ABSTRACT}

After analysing the concept "technical translation" (1) from a double point of view: "technical content" (1.1) and "technical discourse" (1.2), the author presents some basic principles of general methodology applied to "the training of technical translators" (2) such as: employability (2.1), alternation of "dislocation vs a holistic approach" (2.2) and "field representativity" (2.1). In his conclusion, the author suggests some more specific elements of practical methodology (3): professionalism (TRADUTECH and STAGEM) (3.1), visualisation and objective comprehension (3.2) and text/product (3.3).

\section{MOTS-CLÉS/KEYWORDS}

traduction technique, formation, employabilité, spécialisation, terminologie

\section{La notion de "traduction technique»}

La définition de la notion de «traduction technique» passe par un certain nombre d'intermédiaires logiques ou termes (log. ou math.) qui en constituent en quelque sorte les contenus objectifs. Plus concrètement, il y a lieu de situer la traduction technique par rapport à la traduction spécialisée et la traduction scientifique d'un côté et par rapport à un certain nombre d'éléments tels la ou les techniques et la ou les technologies de l'autre.

\subsection{Les «contenus techniques»}

Le point de départ de notre démarche définitoire est constitué par le concept de spécialisation ${ }^{1}$. En effet, toute activité humaine quelle qu'elle soit implique un degré certain de "reconnaissabilité» ou de pertinence et ce, tant sur le plan thématique (les éléments constitutifs) que pragmatique (les conditions et l'environnement communicationnel). Comme exemple, nous citons le domaine de la cuisine et ses innombrables sous-domaines (des appareils ménagers à la vinification en passant par la boulangerie et le marché aux poissons) qui représentent une source permanente et intarissable de formation de «spécialistes» (technostructure). Bien sûr, d'emblée se manifeste 
également l'importance capitale du domaine en tant que «délimiteur» opérationnel et donc comme organe de restriction spécialisant. La banalisation (thématique) et la vulgarisation (pragmatique) en sont les épiphénomènes les plus visibles. La notion de «migraine» se définit ainsi simultanément comme «Douleur intense qui affecte un seul côté de la tête, qui survient sous forme de crises, et s'accompagne de nausées ${ }^{2}$ » et comme «Céphalée primitive vasculaire due a un trouble périodique de la vasomotricité des branches de l'artère carotide externe, d'étiologie mal connue, caractérisée sur le plan clinique par des douleurs paroxystiques, localisées à une moitié de la tête (hémicrânie), des vomissements et parfois accompagnée ou précédée de signes neurologiques $^{3} »$. Le concept de domaine nous permet également d'introduire un second terme dans notre raisonnement qui est l'alternance réalités humaines $v s$ réalités non humaines. Dans ce sens, une traduction juridique est une traduction spécialisée au même titre qu'une traduction technique ou scientifique. Kočourek ${ }^{4}$ distingue dans ce contexte quatre catégories de sciences: les sciences théoriques, les sciences expérimentales, les techniques et la production/consommation. Nous retrouvons pratiquement la même classification chez Bédard ${ }^{5}$ : sciences exactes (théoriques et expérimentales) et techniques. À l'exception de la production/consommation sur laquelle nous reviendrons plus loin, les deux auteurs mentionnés se montrent partisans d'une nouvelle alternance, subordonnée à la catégorie réalités non humaines: sciences exactes (théoriques et expér.) et techniques. Ces deux dernières catégories se distinguent tant par leur objet que par leur méthodologie explicative ou descriptive. Les «techniques» y sont considérées comme "l'ensemble de procédés méthodiques, fondés sur des connaissances scientifiques, employés à la production » plus que comme «la technique» qui elle représente une démarche procédurale plus générale de transformation de la nature. Il s'agit là d'une première restriction presque instrumentale de la notion TT : activité - transformation - production, la deuxième étant celle de la technologie «étude des techniques, des outils, des machines, des matériaux, des composants électroniques » où les «techniques » s'insèrent dans un schéma exclusif de contenus spécifiques et spécifiés (énumérés) dans le sens de "procédés de nature instrumentale ou opérationnelle». C'est encore Bédard qui nous offre la meilleure solution à ce dilemme en prenant en considération des éléments de technologie générale, de technologie appliquée et d'interface industriel. Cette dernière catégorie reprend le concept «production/consommation» de Kočourek et la "production» du dictionnaire général. De par ses contenus spécifiques (contrôle de qualité, service après-vente, pièces de rechange, comptabilité, chaîne de production, régulation et autorégulation, maintenance, entretien, documentation, sécurité, commercialisation, marketing, productivité,...), elle est appelée à assurer le lien entre les deux premières catégories d'éléments citées; la technologie générale et la technologie appliquée, dont la première (comme la mécanique, l'hydraulique, l'électronique et l'électrotechnique) manipule des valeurs techniques communes (p. ex.: force, masse, mouvement, vitesse en mécanique) tandis que les technologies appliquées se rapportent strictement à leur domaine d'application spécifique (p. ex.: la fabrication de papier, le développement de logiciels, la confection de puces électroniques, la fabrication de poudre de lait,...). Nous retrouvons ainsi le même rapport méthodologique que dans le cas de l'alternance sciences-techniques. En conclusion, objectivement, les «contenus techniques» s'articulent dans une triple dynamique: domaine(s) d'activité humaine contenus humains/contenus non humains; contenus non humains - sciences exactes/ 
techniques et techniques - technologie générale/technologie/appliquée/interface industriel. Bien sûr, ce schéma n'est pas fermé et statique. D’une part, il comprend en interne des intersections, des glissements, des absorptions, des chevauchements, des redondances, des «doubles emplois», etc.; d'autre part, il n'exclut aucunement des éléments plus proprement scientifiques et mêmes humains (comme dans la vulgarisation publicitaire). Par ailleurs, cette même «objectivité» est à l'origine d'une évolution au sein même de l'activité traduisante où, d'une part, une langue spécifique (en l'occurrence l'anglais en informatique) se transforme en réalité physique intraduisible (les assembleurs) et où d'autres part le traducteur est amené à manipuler directement l'objet physique de sa traduction (localisation de logiciels, sites Web et produits multimédias). Le texte lui-même devient petit à petit produit. Finalement, cette même objectivité pose un des principes de base de la traduction proprement dite: la compréhension (le niveau de compréhension) du texte/produit source. Le traducteur est-il censé comprendre jusque dans ses moindres détails le texte/produit à traduire? Existe-t-il une compréhension suffisante ou efficace? Pourrait-on parler d'une «compréhension passive» du domaine ${ }^{6}$ ? Quelle est la voie à suivre entre le traducteur spécialisé et le spécialiste traducteur? La réponse à la première question paraît une évidence. En revanche, les trois autres requièrent une réflexion systématique, pédagogique et professionnelle approfondie. Notre point de vue, que nous avons défendu déjà ailleurs, est celui de la terminologie. De même que le traducteur comprend le texte/produit d'origine, le terminologue comprend le terme. Il en saisit le sens (la valeur référentielle) et le «dépassement» (la valeur relationnelle). D’autre part, la terminologie est une discipline de haute valeur pédagogique. Enfin, le terminologue domine par définition la matière et le code, le contenu et le discours.

\subsection{Le "discours technique»}

Le «discours spécialisé» est précisément le résultat de l'intégration des deux faces essentielles de la spécialisation : l'aspect thématique et son implémentation pragmatique ou communicationnelle. Sa catégorisation repose sur une typologie discursive qui intéresse directement la traduction. De même que pour les "contenus », la schématisation du discours n'est jamais absolue. Les concepts de discours spécialisé, discours littéraire, discours scientifique, discours politique, discours publicitaire, discours commercial, discours technique,... coexistent en toute normalité avec des concepts binaires tels que les discours scientifique et technique, discours technico-commercial, discours technico-publicitaire,... Le « discours technique» apparaît ainsi sans un schéma définitoire dynamique (discours spécialisé - discours scientifique; discours scientifique - discours technique/discours scientifique) assorti de nombreux «hybrides» (technicocommercial, technico-juridique,...) rattachés, entre autres, à la composante d'interface industriel.

Il y a cependant un paramètre qui nous paraît fondamental et qui est à la base d'une deuxième typologie discursive particulièrement efficace en matière de techniques: la densité et l'extensibilité terminologiques des éléments pertinents du discours, en d'autres mots des termes qui le sous-tendent et l'intègrent. En effet, dans cet ordre d'idées, nous distinguons le discours terminologique à prépondérance terminologique du discours rédactionnel fondamentalement imprévisible. L'archétype du premier est le catalogue de produits ou le schéma technique; tandis que le deuxième trouve son 
expression la plus parfaite dans le discours littéraire. Il s'agit donc de nouveau d'un paramétrage dynamique qui plutôt que de signaler des contingents définis/mathématiques nous révèle des tendances et surtout des prépondérances. Théoriquement, la densité terminologique est la proportion de termes/non termes calculée sur base des seules unités virtuelles de discours incontestables qui sont les chaînes de caractères. L'extensibilité (syntagmatique) du terme («train d'atterrissage compensé») pose en même temps le problème de sa délimitation et de sa fusion dans le discours (la chaîne de caractères) $)^{7}$.

\section{Formation à la traduction technique: méthodologie générale}

La formation à la traduction technique (plutôt que son enseignement) relève de trois facteurs/paramètres définitoires qui permettent de la situer naturellement dans un processus pédagogique et didactique soit exclusif soit inclusif et surtout de l'adapter utilement aux contenus et aux discours techniques évoqués précédemment. Il s'agit de l'«employabilité», l'alternance "désarticulation vs approche holistique» et la représentativité des domaines.

2.1 La formation à la traduction technique fait partie (ou plutôt fait l'objet) d'une dynamique de formation professionnelle axée sur l'emploi et articulée en six phases successives: les exercices préparatoires, les exercices de simulation, les stages pédagogiques, la certification, les stages d'insertion professionnelle et l'emploi. Les exercices préparatoires sont essentiellement le contrôle qualitatif des pré-requis nécessaires à la formation proprement dite. Les projets de simulation (TRADUTECH) ${ }^{8}$, par contre, et les stages pédagogiques projettent ou placent respectivement les candidats en situation d'emploi réelle. La responsabilité pédagogique reste cependant institutionnelle. Idem pour la certification dont la valeur ou la non-valeur est déterminée par la continuité dans la dynamique pédagogique. Les stages d'insertion professionnelle $(\mathrm{STAGEM})^{9}$ tombent sous la responsabilité des maîtres de stage professionnels dont l'évaluation finale (comme toute évaluation professionnelle) est purement et simplement binaire. Et enfin, l'emploi, point de référence exclusif en matière d'établissement de paramètres/critères de qualité pour tout et chacun des différents niveaux ou secteurs d'évaluation. En conclusion, l'employabilité a un impact direct sur l'organisation de la formation.

2.2 La désarticulation de l'activité traduisante, par contre, n'intervient qu'indirectement dans le processus de formation. La «progression» des candidats à la formation dans leur apprentissage ne passe pas explicitement par une succession d'étapes représentant d'une façon autonome les différents éléments constitutifs de la «traduction technique» (pré-transfert, transfert, post-transfert). Elle s'appuie plutôt sur une dynamique holistique d'intensification (proximité d'emploi) des compétences testées en début de formation (langues, informatique, culture technique, documentation, terminologie,...) et représentées tout au long de la formation par des applications intégrées. La traduction se définit en ces termes de préférence comme une formation intégrée d'application et de spécialisation. Elle trouve sa place idéalement dans des cursus de niveau avancé à orientation linguistique et cognitive (c'est-à-dire 
terminologique) accessible à tous ceux qui remplissent les conditions nécessaires stipulées (et testées) plus haut. En effet, ici encore la terminologie constitue un outil pédagogique particulièrement puissant. De par sa tridimensionnalité pertinente tant pour le discours que pour les contenus techniques que par sa valeur intrinsèquement didactique (la terminologie systématique ou structurée), elle permet de répondre adéquatement à toutes les questions de méthodologie générale de la formation.

2.3 Un troisième facteur concerne la représentativité des domaines. Si nous partons $\mathrm{du}$ principe que le candidat à la formation dispose d'une bonne culture technique générale (y compris une motivation/curiosité pour les «techniques») et qu'il manipule couramment les outils méthodologiques et les procédures lui permettant de «spécialiser»sa culture technique de base en fonction de paramètres stratégiques donnés, le traditionnel et clichématique «choix du domaine» pour la formation se dissipe purement et simplement sous le poids du "besoin de traduction " (de la même façon que nous avons en terminologie le «besoin terminologique»). Par ailleurs, méthodologiquement, tous les domaines permettent de pratiquer, de spécialiser et de parfaire les compétences de base. À condition toutefois d'assurer une approche holistique ciblée emploi et assez riche que pour comprendre les différents aspects du discours et des contenus techniques en traduction (technologie générale, appliquée et interface emploi // terminologie/phraséologie; documentation; outils et techniques de traduction; texte/produit; gestion, organisation et déontologie). Nous voudrions cependant faire une exception pour un domaine qui nous semble particulièrement adéquat, précisément par sa haute représentativité discursive et terminologique de même que par son exceptionnelle importance commerciale: le domaine de l'informatique.

En effet, le domaine de l'informatique rassemble un certain nombre de caractéristiques susceptibles d'intéresser au plus haut point le pédagogue:

1. l'informatique est en même temps technologie générale et technologie appliquée;

2. l'informatique intervient dans de très nombreux autres domaines de spécialisation;

3. l'interface industriel y est particulièrement bien développé;

4. la composante néologique y est omniprésente;

5. le candidat a facilement accès à une documentation typologiquement complète du domaine (revues spécialisées, Internet);

6. disponibilité de matériel didactique de démonstration et de formation;

7. l'informatique est un domaine où le rapport texte/produit a le plus évolué (localisation de logiciels, de sites Web);

8. coïncidence enrichissante des outils du traducteur et l'objet de la traduction.

\section{Formation à la traduction technique : éléments de méthodologie}

Il s'agit en fait de quelques «études de cas » pédagogiques: les projets de formation professionnelle TRADUTECH et STAGEM; la visualisation comme outil de compréhension objective; et le rapport texte-produit. 


\subsection{Deux exemples de la dynamique pédagogique professionnelle citée (2.1): TRADUTECH et STAGEM}

TRADUTECH est un exercice de simulation professionnelle (né comme projet européen: $\mathrm{CME}, \mathrm{CDA}$ et finalement IP) qui se situe entre le stage pédagogique (partie intégrante du cursus) et les exercices pratiques du début de la formation. Il s'agit d'un projet de traduction à distance caractérisé par sa dimension professionnelle (le donneur d'ouvrage, le cahier des charges et l'évaluation finale sont strictement professionnels); son approche holistique (il inclut toutes les «étapes» du processus traduisant: de la réception du texte à traduire jusqu'à la facture, la mise en page et la remise finale); sa dimension européenne (un même texte anglais est traduit simultanément par des étudiants de différents établissements européens dans leur langue maternelle) et, surtout, son fonctionnement par projet (gestion du projet) orienté vers l'apprenant (un étudiant assume la responsabilité du projet). Actuellement, dans la plupart des établissements concernés, il a été officiellement institutionnalisé.

STAGEM (Stage Emploi) est un stage d'insertion professionnelle caractérisé par son aspect anticipatif d'emploi. Il se situe après la certification institutionnelle finale (diplôme,...), tombe sous la responsabilité du maître de stage employeur et est rémunéré. Pendant toute la durée du stage, le stagiaire est suivi par un tuteur/réviseur qui prend la relève du maître de stage pédagogique.

\subsection{Visualisation et compréhension objective}

Un des principe de fonctionnement de base de la traduction technique concerne la compréhension du texte/produit à traduire/adapter. Or, cette compréhension ne s'improvise pas. Elle repose sur une entrée en matière progressive (culture générale culture générale technique - culture technique générale - culture technique spécialisée - compréhension ponctuelle) basée sur un savoir acquis à force de formation et de documentation (information). Il y a cependant un aspect pédagogique qui facilite énormément le processus compréhensif cité: le contact physique, visuel, avec l'objet de la formation/traduction. En fait, le contact peut être direct (avec un objet présent) ou indirect (avec un objet représenté). La normalisation sémiotique moderne nous propose par ailleurs plusieurs typologies de représentation: les images concrètes («konkrete Bilder») vs les représentations graphiques abstraites ( abstrakte graphische Darstellungen»), la représentation graphique ( graphe representation») vs les désignations («designations»). Plus généralement, il s’agit de photos, dessins, schémas, tableaux, diagrammes, plans, symboles, notations, et autres supports nous permettant de mieux saisir non seulement des objets statiques/physiques mais également des processus, des fonctionnements, des modèles, des structures, des avertissements, instructions, interdictions, des mesures, proportions, dimensions et des relations $(\mathrm{H} \text {. Picht })^{10}$. Derrière cette multiplicité de moyens se cache en effet le procédé pédagogique de l'illustration, qui, ne l'oublions pas, peut avoir en terminologie une valeur définitoire certaine. L'illustration sert fondamentalement à éclairer et à établir le lien entre l'objet et toute activité humaine le concernant, fût-ce tout simplement le fait d'y référer par notre activité traduisante. 


\subsection{Texte/produit}

(La formation à) la traduction technique est confrontée de plus en plus à des objets qui ne sont plus de simples «textes» mais des "produits» qui requièrent de la part du traducteur des manipulations d'ordre physique. La traduction de ces "produits», que ce soit de simples codes machine, des données logicielles complexes, des développements multimédias ou des adaptations objectives font partie d'un projet où la part linguistique du travail cède le pas à des opérations techniques qui sont en même temps formelles, objectivement externes (réalisation, implémentation et testing) et internes (localisation). Ici encore, la dynamique citée sous 2.1 et plus particulièrement une pédagogie orientée projet nous paraît la plus adéquate pour la traduction technique.

\section{Conclusion}

En conclusion, les maîtres mots de la (formation à la) traduction technique sont ainsi clairement son degré d'employabilité et la spécialisation. D’abord parce que commercialement il s'agit d'une part essentielle du marché de la traduction en général $^{11}$, mais aussi parce qu'il s'agit d'un type de traduction (contenus et discours) qui représente de la façon la plus intégrale l'activité traduisante proprement dite. L'emploi apparaît donc à deux niveaux : pendant et après la formation. Le lien qui les unit sera la proximité méthodologique établie par le formateur. D’autre part, la formation à la TT représente idéalement une formation de spécialisation qui intervient au moment où les prérequis opérationnels sont déjà parfaitement établis. Elle utilise la terminologie comme outil pédagogique.

\section{NOTES}

1. Le concept de «spécialisation» («traduction spécialisée» et «langue de spécialité ») a toujours été un des concepts clés dans la considération de la traduction technique. Même la terminologie est définie encore aujourd'hui comme «lexique spécifique du domaine» (O. Jiménez Serrano, $L a$ traducción técnica. Inglés-Español. Didáctica y mundo profesional, Granada, Comares, 2002, p. 9: «léxico específico de un campo»). C'est que le concept LSP n'est jamais très loin... Par ailleurs, la terminologie ne saurait se réduire à sa dimension lexicale aussi riche fût-elle ni à un pur ensemble de termes sans aucune forme de loi de composition lui assurant son autonomie d'action systémique.

2. P. Robert, Dictionnaire alphabétique et analogique de la langue française (PR), Paris, 1977, s.v. (dictionnaire général).

3. Dictionnaire médical Masson, Paris, Masson, 1997, p. 653 (dictionnaire spécialisé). Ou encore: "Céphalée intense, généralement hémicrânéenne, le plus souvent orbito-temporale, survenant par crises paroxystiques, fréquemment précédée d'hyperexcitabilité sensorielle (surtout oculaire) et s'accompagnant d'un état nauséeux qui peut aller jusqu'au vomissement» (A. Manuila, Dictionnaire français de Médecine et de Biologie, II, Paris, Masson, 1971, p. 839).

4. R. Kočourek, La langue française de la technique et de la science, Wiesbaden, Brandstetter, 1982-1991, p. 34-37 (éd. de 1991).

6. C. Bédard, La traduction technique: principes et pratique, Montréal, Linguatech, 1986.

7. P. ex: S. Gamero Pérez, La traducción de textos técnicos, Barcelona, Ariel, 2001, p. 42. Voir aussi: H. Marquant, la «compréhension» du texte technique, in Aspects of specialised translation, Paris, La maison du Dictionnaire, Paris, 2001, p. 55-59 et surtout H. Marquant, «Les «connaissances» du traducteur technique», in II Congrés Internacional de Traducció Especialitzada de Barcelona, Barcelona, Universitat Pompeu Eabra, 2002, p. 274.

8. C. Durieux, Fondement didactique de la traduction technique, Paris, Didier, 1988, pose déjà le problème du rapport entre « expression» et «contenu informatif» (p. 23) du «texte technique» (p. 7). D'autre part, elle souligne aussi l'importance pour le «traducteur de textes techniques» de s'intéresser aux 
«usages et emplois» de cette langue thématique (p. 24-25). Ce dernier point a fait l'objet de nombreuses études qualifiées de "phraséologiques» (D. Gouadec, «Nature et traitement des entités phraséologiques", in Terminologie et phraséologie, Paris, La Maison du Dictionnaire, 1994, p. 167-193).

9. Sur TRADUTECH: H. Marquant \& D. Gouadec, «Objectifs de mise en œuvre du projet TRADUTECH», p. 77-81, in D. Gouadec Formation des traducteurs (2). Colloque international Rennes 22-23 septembre 2000, Paris, La maison du Dictionnaire, 2001.

10. STAGEM (STAGE - EMploi) est un projet d'insertion professionnelle co-financé par le FSE (Fonds Social Européen) et la Haute École Léonard de Vinci (Institut Libre Marie Haps) de Bruxelles (20002004). Le problème des stages en traduction (et en terminologie) avait déjà fait l'objet d'un colloque spécialisé en 1981 à l'Université Laval (Québec) (Colloque GIRSTERM-STQ-ACET/Les stages en traduction et en terminologie - 26, 27 et 28 avril 1981, Québec, Université Laval, 1982).

11. H. Picht, «La representación de objetos y conceptos», in Panorama actual de la terminologiá, Gloria Guerrero Ramos-Manuel Fernando Pérez Lagos, Comares, Granada, 2002, p. 275-305

12. O. Jiménez Serrano, La traducción técnica. Inglés-Español: Didáctica y mundo profesional, Granada, Comares, 2002, p. 1; D. Gouadec, Profession Traducteur, La Maison du Dictionnaire, Paris, 2002, p. 89; S. Gamero Pérez, La traducción de textos técnicos, Barcelona, Ariel, p. 17; P. A. Schmitt, Translation und Technik, Tübingen, Stauffenberg, 1999, p. 12-15

\section{RÉFÉRENCES}

BÉDARD, C. (1986) : La traduction technique: principe et pratique, Montréal, Linguatech.

Colloque GIRSTERM - STQ - ACET (1982) : «Les stages en traduction et en terminologie » - 26, 27 et 28 avril 1981, Québec, Université Laval.

Durieux, C. (1988): Fondement didactique de la traduction technique, Paris, Didier.

Gamero Pérez, S. (2001): La traducción de textos técnicos, Barcelona, Ariel.

GouAdec, D. (1994): "Nature et traitement des entités phraséologiques», in Terminologie et phraséologie, Paris, La Maison du Dictionnaire, p. 167-193.

Gouadec, D. (2002): Profession Traducteur, Paris, La Maison du Dictionnaire.

Jiménez Serrano, O. (2002): La traducción técnica. Inglés-Español. Didáctica y mundo profesional, Granada, Comares.

Kočourek, R. (1986-1991): La langue française de la technique et de la science, Wiesdaden, Brandstetter.

Marquant, H. (2001) : «La compréhension du texte technique», in Aspects of specialised translation, Paris, La maison du Dictionnaire, p. 55-59.

Marquant, H. (2002) : «Les “connaissances" du traducteur technique», in II Congrés International de Traducció Especialitzada de Barcelona, Barcelona, Universitat Pompeu Fabra, 271-277.

Marquant, H. et D. Gouadec (2001) : «Objectifs de mise en œuvre du projet TRADUTECH », in Formation des traducteurs (2), Colloque International Rennes 22-23 septembre 2000, Paris, La Maison du Dictionnaire, p. 77-81.

Masson (1997): Dictionnaire médical Masson, Paris, Masson.

Picht, H. (2002) : «La representación de objetos y conceptos», in Panorama actual de la terminología, Granada, Comares, p. 275-305.

Sснмітт, P. A. (1999) : Translation und Technik, Tübingen, Stauffenberg. 